\title{
Normative Study of the Block Design Test for Adults Aged 55 Years and Older in Korean Aging Population
}

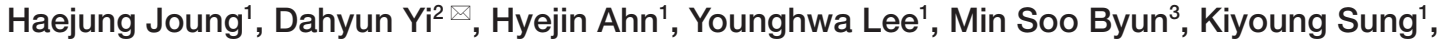 \\ Dongkyun Han', Dong Young Lee ${ }^{1,4,5} \otimes$, and for the KBASE Research Group* \\ 1'Department of Neuropsychiatry, Seoul National University Hospital, Seoul, Republic of Korea \\ ${ }^{2}$ Seoul National University Hospital Biomedical Research Institute, Republic of Korea \\ ${ }^{3}$ Department of Psychiatry, Pusan National University Yangsan Hospital, Yangsan, Republic of Korea \\ ${ }^{4}$ Institute of Human Behavioral Medicine, Medical Research Center Seoul National University, Seoul, Republic of Korea \\ ${ }^{5}$ Department of Psychiatry, Seoul National University College of Medicine, Seoul, Republic of Korea
}

\begin{abstract}
Objective The Block Design Test (BDT) is known to be an effective measure in diagnosing age-related cognitive decline of visuospatial function. The goal of this study is to investigate the effects of age, education years, and gender on the performance of the BDT and to provide normative data in Korean community-dwelling participants who are 55 to 90 years old.

Methods The participants were 432 non-demented adults aging from 55 to 90 years old. The BDT was administered to participants according to its manual. Multiple linear regressions and analyses of variance were conducted, including age, gender, and educations were used as covariates.

Results Age, educational years, and gender were found to be significantly associated with performance on the BDT. As age increased, BDT performance decreased. Educational years were associated with BDT performance. Men showed higher performance (29.9 \pm 10.3$)$ compare to women $(26.1 \pm 8.7)$. The BDT is influenced by age, educational years, and gender.

Conclusion Unlike the previous study, the current study shows that gender has a significant influence in visuospatial ability in the old population. Present normative data will be useful for clinicians in evaluating aging participants with cognitive impairment.
\end{abstract}

Psychiatry Investig

Key Words Block design test, Visuospatial function, Normative data, Aging, Gender differences.

\section{INTRODUCTION}

The Block Design Test (BDT) is a subtest of the Wechsler Adult Intelligence Scale-IV (WAIS-IV) and is a widely used prominent neuropsychological measure in assessing visual spatial ability, constructional praxis, motor skill, and prob-

\footnotetext{
Received: November 26, 2020 Revised: February 2, 2021

Accepted: March 31, 2021

$\triangle$ Correspondence: Dong Young Lee, MD, PhD

Department of Neuropsychiatry, Seoul National University Hospital, 101 Daehak-ro, Jongno-gu, Seoul 03080, Republic of Korea

Tel: +82-2-2072-2205, Fax: +82-2-744-7241, E-mail: selfpsy@snu.ac.kr

$\triangle$ Correspondence: Dahyun Yi, PhD

Seoul National University Hospital Biomedical Research Institute, 101 Daehakro, Jongno-gu, Seoul 03080, Republic of Korea

Tel: +82-2-2072-4586, Fax: +82-2-744-7241, E-mail: dahyunyi@gmail.com

*The co-investigators of the KBASE Research Group are listed in elsewhere (http://kbase.kr).

(c) This is an Open Access article distributed under the terms of the Creative Commons Attribution Non-Commercial License (https://creativecommons.org/licenses/by$\mathrm{nc} / 4.0$ ) which permits unrestricted non-commercial use, distribution, and reproduction in any medium, provided the original work is properly cited.
}

lem-solving skill ability. ${ }^{1-4}$ The BDT is considered the most sensitive of the WAIS-R subtests to observe central nervous system dysfunction ${ }^{1,5}$ and is used particularly for evaluating changes in cognitive status between being cognitively healthy, with Mild Cognitive Impairment (MCI), or in dementia. ${ }^{6}$ The performance is a timed measure for visuospatial constructional ability. The BDT requires performer to arrange a set of four or nine blocks with different colored sides to duplicate a maximum of fourteen patterns presented in ascending order of difficulty with time limitation. Korean version of BDT WAIS-IV was used, which contains the same stimuli and follows the same procedures as original WAIS-IV version.

The normative data for the BDT was obtained from Caucasian residing in the United State who are between 16 to 74 years old, ${ }^{1}$ with educational attainment ranging from 7 years to college or more. The BDT has been known to be highly related to the demographic factors; age, education, gender, race, occupation and cultural background. ${ }^{3,6-15}$ Previous BDT norma- 
tive data did not report gender differences. ${ }^{6,8,10,12,15,16}$ Yet, there are consistent findings on gender effects on visuospatial neuropsychological testing, generally reporting better performance by men. ${ }^{17-19}$ Gender effects on the BDT were also reported consistently, showing men outperforming women., ${ }^{7,20-22}$ Despite consistent reports of the effects of gender on visuospatial test performance, previous BDT normative data do not incorporate gender effects.

Consideration and incorporation of gender difference when developing normative data is particularly important for the Korean adults because of different educational and/or occupational opportunities depending on gender for the aging population in South Korea. Therefore, consideration of gender effect is necessary in addition to the effects of education. Inappropriate application of normative data that dismiss the cultural factors may pose a major challenge to clinical research and clinicians. Taken together, we aim to report on the joint effects of age, education and gender demographic variable on BDT performance in non-demented aging population and to provide normative data.

\section{METHODS}

\section{Study population}

This study included 432 non-demented Korean between 55-90 years old. Research participants were recruited from an ongoing prospective cohort study, Korean Brain Aging Study for Early diagnosis and prediction of Alzheimer's Disease (KBASE). All participants underwent comprehensive clinical and neuropsychological evaluations. Psychiatrists with advanced training in neuropsychiatry and dementia research; individually examined all participates using the clinical assessment batter in the Korean Version of the Consortium to Establish Registry for Alzheimer's Disease (CERAD). ${ }^{23}$ The battery consists of a standardized clinical interview on demographic information, cognitive and functional status, drug inventory, depression and medical history, a cognitive state examination and a general physical and neurological examination. Reliable informants were also interviewed to acquire accurate information regarding the cognitive and functional changes and medical history of participants. ${ }^{24}$

Older adults with normal cognition (NC) or mild cognitive impairments (MCI) were analyzed. The inclusion criteria for NC were: 1 ) age 55-90 years (inclusive), 2) CDR score of 0 , and 3) no diagnosis of MCI or dementia. Secondly, individuals who met the following inclusion criteria based on core clinical criteria for diagnosis of MCI according to the recommendations of the NIA-AA guidelines were included: 1) age 55-90 years (inclusive), 2) memory complaint corroborated by self, an informant, or clinician, 3) objective memory impairment for age, education, and gender, 4) largely intact functional activities, and 5) not demented.

Participants with dementia and other serious medical psychiatric and neurological disorders that could affect mental function were excluded from the analysis. A diagnosis of dementia was made according to the criteria of the fourth edition of the Diagnostic and Statistical Manual of Mental Disorders (DSM-IV) American Psychiatric. ${ }^{25}$ Participants who successfully completed whole neuropsychological tests are included in the current study. This study was ethically approved by Seoul National University/Seoul National University Hospital Intuitional Review Board (IRB No. H-2006-1011132).

\section{Measurement}

\section{WAIS-IV-K Block Design Test}

The BDT used in this stud is from Korean Wechsler Adult Intelligence Scale, 4th Edition (WAIS-IV-K) ${ }^{26}$ It involves subjects to reconstruct the two-dimensional designs using either four or nine, red and white colored blocks. Each trial is timed, and bonus points are given for faster completion. Scores on the BDT range from 0 to 48 , with bonus points up to 66 . The higher score reflects better visuospatial functioning. In this study, BDT performance was scored in the standard manner; we used the bonus timed score of the test for the performance analysis.

\section{Statistical analysis}

Statistical analyses were conducted with SPSS (version 24.0; Statistical Package for the Social Sciences for Window; IBM Corp, Armonk, NY, USA). A stepwise multiple linear regression analysis was performed to assess the influence of age, educational years and gender on BDT score. Age and educational years were entered as continuous variables and gender was coded as 1 or 0 for man or women. A series of $2 \times 5 \times 2$ analyses of variance (ANOVA) were also performed to determine the main effect of age (55-74 and 75-90 years), education ( 0 $3,4-6,7-9,10-12$, and $\leq 13$ ), and gender (women versus men) on the test performance. Post hoc contrasts with Tukey's method were conducted when the main effects were significant at the $\mathrm{p}<0.05$ level.

\section{RESULTS}

\section{Demographic characteristic}

The demographic characteristics of participant who completed the BDT are presented in Table 1. The mean age of the participants was 70.8 years $(\mathrm{SD}=8.0)$ and ranged from 55 to 90 years. $56 \%$ were composed of women. The average score of 
Table 1. Demographic characteristics of the participants

\begin{tabular}{cccc}
\hline & Men $(\mathrm{N})$ & Women $(\mathrm{N})$ & Total $(\mathrm{N})$ \\
\hline Age $(\mathrm{yr})$ & $190(70.8 \pm 8.4)$ & $242(70.4 \pm 7.7)$ & $432(70.5 \pm 8.0)$ \\
$55-64$ & $54(60.1 \pm 2.7)$ & $57(59.5 \pm 2.5)$ & $111(59.8 \pm 2.6)$ \\
$65-69$ & $24(67.2 \pm 1.5)$ & $51(67.2 \pm 1.3)$ & $75(67.2 \pm 1.4)$ \\
$70-74$ & $45(72.3 \pm 1.4)$ & $43(72.0 \pm 1.4)$ & $88(72.2 \pm 1.4)$ \\
$75-79$ & $34(76.5 \pm 1.4)$ & $63(76.5 \pm 1.3)$ & $97(76.5 \pm 1.3)$ \\
$80-90$ & $33(82.9 \pm 2.8)$ & $28(81.9 \pm 2.1)$ & $61(82.4 \pm 2.5)$ \\
Education (yr) & $13.1 \pm 4.2$ & $9.7 \pm 4.7$ & $11.2 \pm 4.8$ \\
MMSE & $26.2 \pm 3.2$ & $24.8 \pm 3.6$ & $25.4 \pm 3.5$ \\
BDT & $29.9 \pm 10.3$ & $26.1 \pm 8.7$ & $27.8 \pm 9.6$ \\
\hline
\end{tabular}

(Mean \pm SD). SD: standard deviation, MMSE: mini mental standardized exam, BDT: block design test

Table 2. Stepwise multiple linear regression of age, educational years and gender on the BDT

\begin{tabular}{lcccc}
\hline \multicolumn{1}{c}{ Variables } & $\mathrm{B}$ & $\mathrm{SE}(\mathrm{B})$ & $\beta$ & $\Delta \mathrm{R}^{2}$ \\
\hline BDT & & & & \\
Education & 0.629 & 0.091 & $0.311^{\dagger}$ & 0.156 \\
Age & -0.386 & 0.051 & $-0.322^{\dagger}$ & 0.096 \\
Gender & 1.803 & 0.863 & $0.093^{*}$ & 0.008 \\
\hline
\end{tabular}

Age and educational years were entered as continuous variables, and gender was coded as 0 and 1 for woman and man, respectively. ${ }^{*} \mathrm{p}<0.01,{ }^{\dagger} \mathrm{p}<0.001$. B: regression coefficient, $\mathrm{SE}(\mathrm{B})$ : standard error of $B, \beta$ : standardized regression coefficient, $\Delta R^{2}$ : percent variance explained by each variable, BDT: block design test

the men on the BDT was $29.9(\mathrm{SD}=10.3)$ and the women average score was $26.1(\mathrm{SD}=8.7)$. There was a significant difference between men $(\mathrm{M}=13.1, \mathrm{SD}=4.17)$ and women $(\mathrm{M}=9.7$, $\mathrm{SD}=4.67)$ in educational years $[\mathrm{F}(1,430)=61.4, \mathrm{p}<0.001]$ and BDT score $[\mathrm{F}(1,430)=17.02, \mathrm{p}<0.001]$.

\section{Effect of age, education, and gender on BDT score}

Stepwise regression analysis showed age, education and gender showed significant main effects by each variable on BDT score. Age $[\mathrm{F}(1,431)=67.36, \mathrm{p}<0.001]$, education $[\mathrm{F}(1,431)=79.28$, $\mathrm{p}<0.001]$ and gender $[\mathrm{F}(1,431)=17.03, \mathrm{p}<0.001]$ were found to independently have significant effects on BDT scores. There was significant interactions between gender and education $[\mathrm{F}(1,431)=61.37, \mathrm{p}<0.001]$ and between age and education $[F(1,431)=10.75, p<0.001]$ were found to have significant effects on BDT scores. Age and gender interaction effect was not significant $[\mathrm{F}(1,431)=17.54, \mathrm{p}=0.602]$.

Among the variables, education explained the largest variance of BDT performance (Table 2). To see the education effect of the sub groups (ie, $0-3,4-6,7-9,10-12$, and $\leq 13$ years) were compared using post hoc contrasts. There were no significant differences between $0-3$ with $4-6$, and 7-9 with $10-12$ years group. Therefore, groups were divided into three groups
$(0-6,7-12$, and $\leq 13$ years). Similar to the previous studies, age also significantly affected BDT performance; ${ }^{13,14}$ as age increased the performance of BDT declined significantly. Also, ANOVA was performed $[F(1,430)=20.9, p<0.001]$ to identity the differences between age groups.

\section{Normative date of the BDT}

Stepwise multiple linear regression analyses revealed that, age, education years, and gender significantly predicted BDT scores. Tables 3 and 4 shows the normative data of each age group according to the level of education and gender. Given that BDT performance is influenced by age, educational levels and gender, participants were divided into five age overlapping stratification groups for the normative data. The over overlapping strata was used to maximize the clinical usefulness of normative test data. ${ }^{27}$ Tables composed of overlapping age strata with midpoint ages occurring at 5-year intervals (ie, $67,72,77$, and 82 years of age, respectively for four strata) were developed as shown in Tables 3 and 4. In each table, the user should select the age strata with a midpoint closest to the subject's age to interpret $\mathrm{BDT}$ performance. As a result, normative data from ages $55-64,60-74,65-79,70-84$ and $75-90$ years were used for persons whose ages ranged from $55-64,70-74$, $75-79,80-90$ years, respectively. Each of these ranges was derived to encompass all ages closer to one midpoint age than to the other adjacent midpoints. Educational groups were divided into three strata (ie, $0-6,7-12$, and $\leq 13$ years of education).

\section{DISCUSSION}

The present study was conducted to examine the effect of demographic factors on the performance on the BDT and to provide normative data for middle to older aged adults in an educationally diverse population with consideration of the effects of gender, which is anticipated to better assist clinicians and researchers when making interpretation of the test performance. ${ }^{17,20,22,28}$ The result showed age, education, and gender were independently associated with BDT performance.

In the present study, BDT performance declined with age, which is consistent with previous findings. ${ }^{6,714}$ The results of age-related decline in BDT indicate that visuospatial ability declines with normal aging. Educational years were associated with better performance on the BDT and lower educational years were associated with poorer performance. Our finding is consistent with other normative studies that showed a significant association between education and BDT performance. ${ }^{7-10,12,13}$ Moreover, men performed better than women on visuospatial tests independent of years of education. There was also significant interaction effects of gender and education on BDT (Figure 1). 
The findings on age and education are consistent with those reported in other normative studies. The contribution of gender to the BDT performance found in our study, however, was

Table 3. Men normative data on the BDT stratified by age and education; mean, standard deviation, median and rage from the 5th to 95th percentile

\begin{tabular}{|c|c|c|c|c|}
\hline Education (yr) & $0-6$ & $7-12$ & $\leq 13$ & Total \\
\hline \multicolumn{5}{|l|}{ Age (yr) } \\
\hline \multicolumn{5}{|l|}{$55-64^{*}$} \\
\hline $\mathrm{N}$ & & 22 & 31 & 53 \\
\hline Mean & & 31.73 & 41.13 & 37.23 \\
\hline SD & & 10.375 & 10.948 & 11.597 \\
\hline Median & & 28 & 43 & \\
\hline 5th percentile & & 27.13 & 37.11 & 34.03 \\
\hline 95th percentile & & 36.33 & 45.14 & 40.42 \\
\hline \multicolumn{5}{|l|}{$65-69^{\dagger}$} \\
\hline $\mathrm{N}$ & 11 & 41 & 48 & 100 \\
\hline Mean & 25.64 & 28.66 & 32 & 29.93 \\
\hline SD & 8.346 & 9.076 & 11.248 & 10.256 \\
\hline Median & 24 & 27 & 31 & 28 \\
\hline 5 th percentile & 20.03 & 25.79 & 28.73 & 27.9 \\
\hline 95th percentile & 31.24 & 31.52 & 35.27 & 31.96 \\
\hline \multicolumn{5}{|l|}{$70-74^{\ddagger}$} \\
\hline $\mathrm{N}$ & 17 & 37 & 49 & 103 \\
\hline Mean & 25.29 & 27 & 28.94 & 27.64 \\
\hline SD & 6.678 & 8.4 & 9.038 & 8.498 \\
\hline Median & 24 & 25 & 29 & 28 \\
\hline 5th percentile & 21.86 & 24.2 & 26.34 & 25.98 \\
\hline 95th percentile & 28.73 & 29.8 & 31.53 & 29.3 \\
\hline \multicolumn{5}{|l|}{$75-79 \S$} \\
\hline $\mathrm{N}$ & 17 & 34 & 52 & 103 \\
\hline Mean & 24.18 & 26.29 & 27.27 & 26.44 \\
\hline SD & 7.187 & 6.313 & 8.525 & 7.651 \\
\hline Median & 24 & 25.5 & 25.5 & 24 \\
\hline 5th percentile & 20.48 & 24.09 & 24.9 & 24.94 \\
\hline 95th percentile & 27.87 & 28.5 & 29.64 & 27.93 \\
\hline \multicolumn{5}{|l|}{$80-90^{\| \prime}$} \\
\hline $\mathrm{N}$ & 10 & 20 & 34 & 64 \\
\hline Mean & 24 & 24.9 & 27.5 & 26.14 \\
\hline $\mathrm{SD}$ & 5.754 & 6.735 & 5.701 & 6.136 \\
\hline Median & 24 & 24 & 27 & 24 \\
\hline 5th percentile & 19.88 & 21.75 & 25.51 & 24.61 \\
\hline 95th percentile & 28.12 & 28.05 & 29.49 & 27.67 \\
\hline
\end{tabular}

*normative data from the people whose ages range from 55 to 64 , tnormative data from the people whose ages range from 60 to 74, ‡Normative data from the people whose ages range from 65 to 79 , $\S$ Normative data from the people whose ages range from 70 to 84 , "Normative data from the people whose ages range from 76 to 90 . SD: standard deviation, BDT: block design test unique from that of previous studies, ${ }^{6,8,12}$ given that the previous normative studies on BDT either reported non-significant gender effects or chose to not consider the effects of gender

Table 4. Women normative data on the BDT stratified by age and education; mean, standard deviation, median and rage from the 5th to 95 th percentile

\begin{tabular}{|c|c|c|c|c|}
\hline Education (yr) & $0-6$ & $7-12$ & $\leq 13$ & Total \\
\hline \multicolumn{5}{|l|}{ Age (yr) } \\
\hline \multicolumn{5}{|l|}{$55-64^{*}$} \\
\hline $\mathrm{N}$ & & 30 & 16 & 46 \\
\hline Mean & & 29.17 & 38.13 & 32.28 \\
\hline SD & & 6.029 & 10.62 & 8.924 \\
\hline Median & & 29 & 40 & \\
\hline 5 th percentile & & 26.92 & 32.47 & 29.63 \\
\hline 95th percentile & & 31.42 & 43.78 & 34.93 \\
\hline \multicolumn{5}{|l|}{$65-69^{\dagger}$} \\
\hline $\mathrm{N}$ & 37 & 60 & 22 & 119 \\
\hline Mean & 22.3 & 27.57 & 27.82 & 25.97 \\
\hline SD & 8.749 & 7.115 & 7.694 & 8.085 \\
\hline Median & 20 & 28 & 24 & 24 \\
\hline 5 th percentile & 19.38 & 25.73 & 24.41 & 24.51 \\
\hline 95th percentile & 25.21 & 29.4 & 31.23 & 27.44 \\
\hline \multicolumn{5}{|l|}{$70-74^{\ddagger}$} \\
\hline $\mathrm{N}$ & 59 & 68 & 30 & 157 \\
\hline Mean & 22.19 & 26.21 & 27.83 & 25.01 \\
\hline SD & 7.675 & 7.179 & 7.931 & 7.804 \\
\hline Median & 24 & 25 & 24 & 24 \\
\hline 5th percentile & 20.19 & 24.47 & 24.87 & 23.78 \\
\hline 95th percentile & 24.19 & 27.94 & 30.79 & 26.24 \\
\hline \multicolumn{5}{|l|}{$75-79 \S$} \\
\hline $\mathrm{N}$ & 54 & 52 & 24 & 130 \\
\hline Mean & 22.06 & 25.31 & 29.13 & 24.66 \\
\hline SD & 7.383 & 7.643 & 7.881 & 7.955 \\
\hline Median & 24 & 26.5 & 28 & 24 \\
\hline 5 th percentile & 20.04 & 23.18 & 25.8 & 23.28 \\
\hline 95th percentile & 24.07 & 27.44 & 32.45 & 26.04 \\
\hline \multicolumn{5}{|l|}{$80-90^{\prime \prime}$} \\
\hline $\mathrm{N}$ & 40 & 35 & 16 & 91 \\
\hline Mean & 21.45 & 25.46 & 28.13 & 24.16 \\
\hline SD & 6.421 & 7.613 & 8.041 & 7.566 \\
\hline Median & 24 & 28 & 26 & 24 \\
\hline 5th percentile & 19.4 & 22.84 & 23.84 & 22.59 \\
\hline 95th percentile & 23.5 & 28.07 & 32.41 & 25.74 \\
\hline
\end{tabular}

*normative data from the people whose ages range from 55 to 64 , tnormative data from the people whose ages range from 60 to 74,

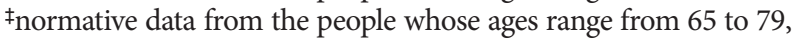
\$normative data from the people whose ages range from 70 to 84 , "normative data from the people whose ages range from 76 to 90. SD: standard deviation, BDT: block design test 


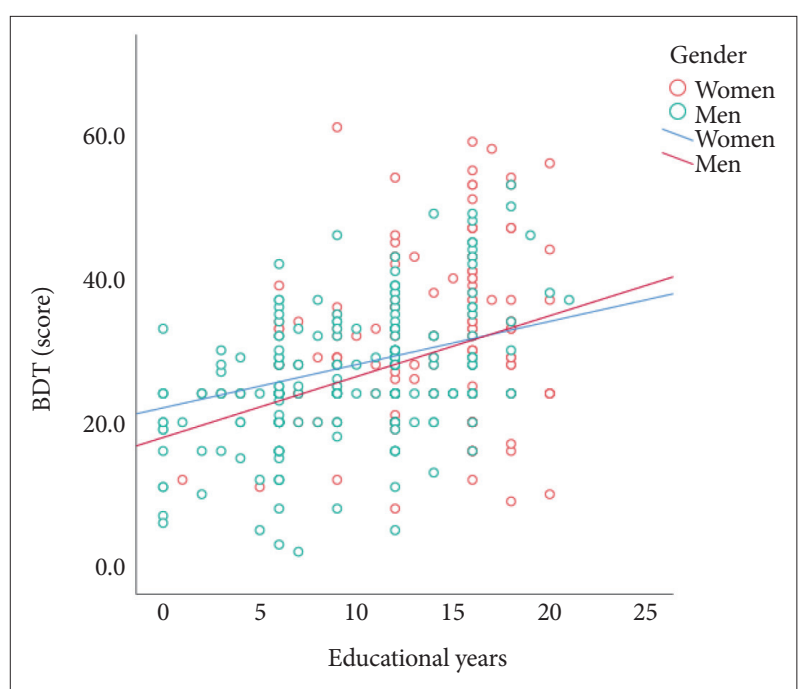

Figure 1. Gender and educational level interaction on the BDT performance. BDT: block design test, Gender 0: men, 1: women.

due to sample size. Korean older adults have significantly different educational attainment by gender, which is reflected in the results. This may be above and beyond the implicated underlying biological differences of gender. It has been speculated that the differences in neuropsychological performance between genders, especially in the old population, may be due to the reduced education, employment, and social engagement in women in less developed countries in the past. The present study supports the earlier studies of men having higher performance in visuospatial ability tests. ${ }^{3,15,20,22,29}$ Notably, higher educational attainment of men might be related with intellectual activates during a lifetime, leading to the protection of aging individuals against cognitive decline possibly through increased intellectual activities as well as better economic resources to protect against dementia. ${ }^{6,14,30}$

Gender effects shown in the current study compared to previous reports may appear to be amplified for Asians-particularly South Koreans. Previous studies suggested influence of not only gender and socio-economic factors but also ethnicity on educational attainment. ${ }^{30,31}$ In other words, understanding of the effects of education on cognitive function of elderly living in South Korea cannot be separate from the effects of gender. Furthermore, the difference in the educational attainment levels leads to discrepant future employment prospects and access to opportunities. Such gender-based disparities are notable and reflected in this normative study. Many older women in South Korea were required to tend to household duties from a very young age to older adulthood and were often not supported to attend schools. It was considered that women's role in life was to support men and their children. Men, on the other hand, had more opportunities to work outside, to have social roles, and be more independent. ${ }^{32}$ Even with less or unschool- ing, men had greater opportunities working outside the home environment and interacting with others as a primary income earner of the family, which enabled men but not women to strengthen cognitive or literacy skills. Consequently, less educated men were able to educate themselves through exposure to diverse or stimulating environment, which was not the case for women. The effect of such disparity disappears in the age cohorts where women received equal or similar education as men, such as shown in the current normative data. The cultural or ethnic differences can be observed by the similar social experiences when the gender effect is lessened to a significant degree. ${ }^{8,33}$ These previous studies were mostly made up of the Caucasian population. Although women may serve a similar social-economic role in society, their educational attainments were higher, leading to results have less significant differences between men and women. ${ }^{33}$

There is a limitation that should be acknowledged. The participants who are young (between 55-64 years of age) and less educated (between 0-6 years of education) are limited in size, which is due to changes in societal and educational system in South Korea in recent decades. If possible, future studies including middle-aged adult participants with various educational levels may be helpful.

In conclusion, taking together the findings from the analyses, we developed the normative data to adjust for age, gender, education factors for BDT. By adopting the overlapping age stratification method proposed by Pauker, ${ }^{27}$ we were able to present more diverse and stable normative data of older adults with a sufficient number of participants in each normative block. This study provides demographically well-adjusted normative data for BDT score for a large diverse and rapidly growing population. Because earlier normative data for BDT were mostly based on a well-educated population, this study provides normative data for the educationally diverse sample and, therefore, offers more accurate information that will be more helpful in determining the severity of cognitive decline due to aging.

\section{Acknowledgments}

This study was supported by a grant from the Ministry of Science and ICT, Republic of Korea (Grant No. NRF-2014M3C7A1046042) and a grant of the Korea Health Technology R\&D Project through the Korea Health Industry Development Institute (KHIDI) funded by the Ministry of Health \& Welfare, Republic of Korea (grant number: HI18C0630 \& HI19C0149).

\section{Conflicts of Interest}

The authors have no potential conflicts of interest to disclose.

\section{Author Contributions}

Conceptualization: Dong Young Lee, Dahyun Yi, Haejung Joung. Data curation: Haejung Joung. Formal analysis: Haejung Joung. Funding acquisition: Dong Young Lee. Investigation: Haejung Joung, Young Hwa Lee, Hyejin Ahn, Kiyoung Sung, Dongkyun Han, Min Soo Byun, Dahyun Yi. 
Methodology: Haejung Joung, Young Hwa Lee, Hyejin Ahn, Kiyoung Sung, Dongkyun Han, Min Soo Byun, Dahyun Yi. Project administration: Haejung Joung, Young Hwa Lee, Hyejin Ahn, Kiyoung Sung, Dongkyun Han, Min Soo Byun, Dahyun Yi. Resources: Haejung Joung, Young Hwa Lee, Hyejin Ahn, Kiyoung Sung, Dongkyun Han, Min Soo Byun, Dahyun Yi. Supervision: Dong Young Lee, Dahyun Yi. Validation: Dong Young Lee, Dahyun Yi. Visualization: Haejung Joung, Dahyun Yi. Writing-original draft: Haejung Joung. Writing_-review \& editing: Dong Young Lee, Dahyun Yi, Haejung Joung.

\section{ORCID iDs}

\section{Haejung Joung}

Dahyun Yi

Hyejun Ahn

Younghwa Lee

Min Soo Byun

Kiyoung Sung

Dongkyun Han

Dong Young Lee https://orcid.org/0000-0002-2617-7758 https://orcid.org/0000-0002-0860-0946 https://orcid.org/0000-0002-6613-4680 https://orcid.org/0000-0002-5312-212X https://orcid.org/0000-0003-3159-4510 https://orcid.org/0000-0002-7552-2888 https://orcid.org/0000-0002-5245-1028 https://orcid.org/0000-0001-8976-8320

\section{REFERENCES}

1. Wechsler D. WAIS-R Manual: Wechsler Adult Intelligence Scale-revised. New York: Psychological Corporation; 1981.

2. Lazarević LB, Knežević G, Mitić M, Jočić DD. Psychometric properties of the Serbian version of the Wechsler adult intelligence Scale-Fourth Edition (WAIS-IV). Psihologija 2018;51:333-349.

3. Kaufman AS, McLean JE, Reynolds CR. Sex, race, residence, region, and education differences on the 11 WAIS-R subtests. J Clin Psychol 1988;44:231-248.

4. Valentine T, Block C, Eversole K, Boxley L, Dawson E. Wechsler Adult Intelligence Scale-IV (WAIS-IV). In: Carducci BJ, Editor. The WileyBlackwell Encyclopedia of Personality and Individual Differences. Hoboken, NJ: John Wiley \& Sons, 2018, p.457-463.

5. Lezak MD. Neuropsychological Assessment, 3rd Ed. New York, NY, US: Oxford University Press; 1995.

6. Yin S, Zhu X, Huang X, Li J. Visuospatial characteristics of an elderly Chinese population: results from the WAIS-R block design test. Front Aging Neurosci 2015;7:17.

7. Ponton MO, Satz P, Herrera L, Ortiz F, Urrutia CP, Young R, et al. Normative data stratified by age and education for the Neuropsychological Screening Battery for Hispanics (NeSBHIS): Initial report. J Int Neuropsychol Soc 1996;2:96-104.

8. Elias MF, Dore GA, Goodell AL, Davey A, Zilioli MK, Brennan S, et al. Normative data for elderly adults: the Maine-Syracuse study. Exp Aging Res 2011;37:142-178.

9. Elkadi S, Clark MS, Dennerstein L, Guthrie JR, Bowden SC, Henderson VW. Normative visuospatial performance in Australian midlife women. Aust Psychol 2006;41:43-47.

10. Ivnik RJ, Malec JF, Smith GE, Tangalos EG, Petersen RC, Kokmen E, et al. Mayo's older americans normative studies: WAIS-R norms for ages 56 to 97. Clin Neuropsychol 1992;6:1-30.

11. Zink DN, Miller JB, Caldwell JZK, Bird C, Banks SJ. The relationship between neuropsychological tests of visuospatial function and lobar cortical thickness. J Clin Exp Neuropsychol 2018;40:518-527.

12. Ganguli M, Snitz BE, Lee CW, Vanderbilt J, Saxton JA, Chang CC. Age and education effects and norms on a cognitive test battery from a population-based cohort: the Monongahela-Youghiogheny Healthy Aging Team. Aging Ment Health 2010;14:100-107.

13. Joy S, Fein D, Kaplan E, Freedman M. Quantifying qualitative features of Block Design performance among healthy older adults. Arch Clin Neuropsychol 2001;16:157-170.
14. Wahlin TBR, Backman L, Wahlin A, Winblad B. Visuospatial functioning and spatial orientation in a community-based sample of healthy very old persons. Arch Gerontol Geriatr 1993;17:165-177.

15. Wang Q, Sun J, Ma X, Wang Y, Yao J, Deng W, et al. Normative data on a battery of neuropsychological tests in the Han Chinese population. J Neuropsychol 2011;5:126-142.

16. Rönnlund M, Nilsson LG. Adult life-span patterns in WAIS-R Block Design performance: Cross-sectional versus longitudinal age gradients and relations to demographic factors. Intelligence 2006;34:63-78.

17. Diaz RR, Lynn R. Sex differences on the WAIS-IV in Chile. Mankind Q 2016;57:52-57.

18. Heaton RK, Ryan L, Grant I, Matthews CG. Demographic Influences on Neuropsychological Test Performance. In: Grant I, Adams KM. Editors. Neuropsychological Assessment of Neuropsychiatric Disorders (2nd Edition). New York: Oxford University Press, 1996, p.141-163.

19. Polunina A, Bryun E, Sydniaeva E, Golukhova E. Gender differences in cognitive functions: retrospective analysis of the data of 5 neuropsychological studies. Sch Rep 2018;3:61-75.

20. de Frias CM, Nilsson LG, Herlitz A. Sex differences in cognition are stable over a 10-year period in adulthood and old age. Neuropsychol Dev Cogn B Aging Neuropsychol Cogn 2006;13:574-587.

21. Parsons TD, Rizzo AR, van der Zaag C, McGee JS, Buckwalter JG. Gender differences and cognition among older adults. Aging Neuropsychol Cogn 2005;12:78-88.

22. Weiss E, Siedentopf CM, Hofer A, Deisenhammer EA, Hoptman MJ, Kremser C, et al. Sex differences in brain activation pattern during a visuospatial cognitive task: a functional magnetic resonance imaging study in healthy volunteers. Neurosci Lett 2003;344:169-172.

23. Lee DY, Lee KU, Lee JH, Kim KW, Jhoo JH, Kim SY, et al. A normative study of the CERAD neuropsychological assessment battery in the Korean elderly. J Int Neuropsychol Soc 2004;10:72-81.

24. Byun MS, Yi D, Lee JH, Choe YM, Sohn BK, Lee JY, et al. Korean brain aging study for the early diagnosis and prediction of Alzheimer's disease: methodology and baseline sample characteristics. Psychiatry Investig 2017;14:851-863.

25. American Psychiatric Association. Diagnostic and Statistical Manual of Mental Disorders: DSM-IV-TR. Washington, DC: American Psychiatric Assocation Inc.; 2000.

26. Hwang S, Kim J, Park K, Chey J, Hong S. Korean Wechsler Adult Intelligence Test (4th Ed.). Daegu: Korean Psychology; 2011.

27. Pauker JD. Constructing overlapping cell tables to maximize the clinical usefulness of normative test data: rationale and an example from neuropsychology. J Clin Psychol 1988;44:930-933.

28. Laws KR, Irvine K, Gale TM. Sex differences in cognitive impairment in Alzheimer's disease. World J Psychiatry 2016;6:54-65.

29. Thilers PP, Macdonald SW, Herlitz A. The association between endogenous free testosterone and cognitive performance: a population-based study in 35 to 90 year-old men and women. Psychoneuroendocrinology 2006;31:565-576.

30. Sammons P. Gender, Ethnic and Socio-economic Differences in Attainment and Progress: a longitudinal analysis of student achievement over 9 years. Br Educ Res J 1995;21:465-485.

31. Sahadevan S, Tan NJ, Tan T, Tan S. Cognitive testing of elderly Chinese people in Singapore: influence of education and age on normative scores. Age Ageing 1997;26:481-486.

32. Yang L, Unverzagt FW, Jin Y, Hendrie HC, Liang C, Hall KS, et al. Normative data for neuropsychological tests in a rural elderly Chinese cohort. Clin Neuropsychol 2012;26:641-653.

33. Malec JF, Ivnik RJ, Smith GE, Tangalos EG, Petersen RC, Kokmen E, et al. Mayo's older americans normative studies: Utility of corrections for age and education for the WAIS-R. Clin Neuropsychol 1992;6(Suppl): $31-47$. 